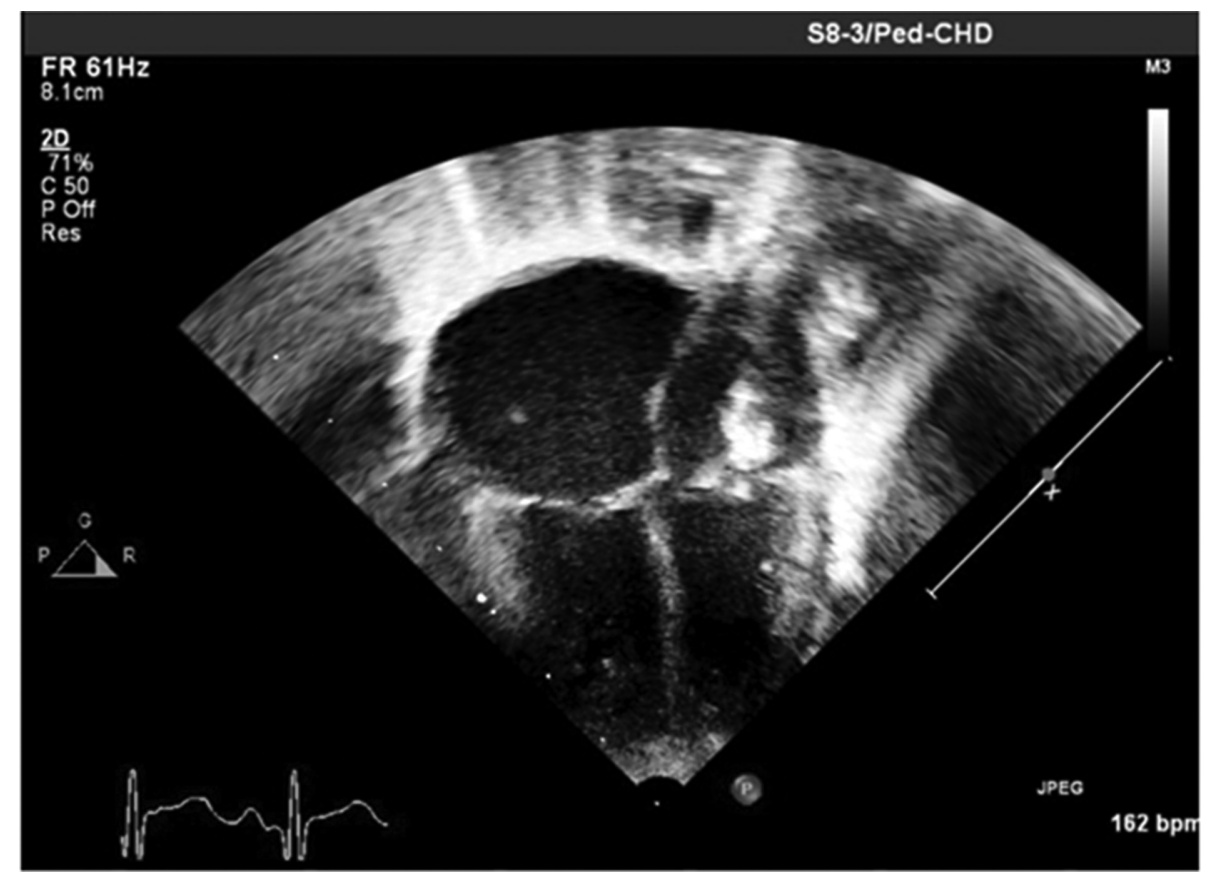

Abstract G464(P) Figure 1 Four chamber view on 2D echocardiogram showing mobile, echogenic vegetations measuring $9.2 \times 3.6 \mathrm{~mm}$, and $3.8 \times 3.2 \mathrm{~mm}$ on leaflets of the mitral valve, bulging into left atrium and left ventricle

recurrent thrombus formation requiring valve replacement. He is currently stable on full anticoagulation therapy.

Conclusion Our case highlights the aggressive nature of GBS with potentially fatal complications requiring a high index of suspicion, as only with early recognition and management of endocarditis can morbidity and mortality be improved. We recommend that repeated parental concern in the context of failing to thrive, lethargy or fever should alert health professionals to the possibility of sepsis.

Although Screening for GBS is not recommended by the UK National Screening Committee nor the Royal College of Obstetricians and Gynaecologists, it has a high profile in the United States where the Centres for Disease Control and Prevention recommends routine screening for all pregnant women, and we suggest this should be so in the UK.

\section{G465(P) THE ROLE OF THE SCHOOL DOCTOR TODAY: AN ADVOCATE FOR CHILDREN WITH OBESITY}

V Impey, M Buckingham, A Lorek. Community Paediatrics, Guy's and St Thomas' NHS Trust, London, UK

\subsection{6/archdischild-2015-308599.419}

Aims To evaluate school doctor service with regard to weight problems in a Secondary School for students with special needs Background School medicals are only offered based on teacher's concerns and there was no existing health screen of potential need.

The school doctor was concerned about potential unaddressed weight problems.

Methods All students were offered a medical by opt-out permission letters to parents, also asking about concerns (diet, sleep, behaviour or other).

Teachers were asked to identify students they felt had an unhealthy BMI.
All students consenting were measured by multidisciplinary team (School doctors, School Nurse, Special Needs specialist dietician, Specialist nurse for obesity).

We calculated BMI centile on WHO growth charts.

An action plan was developed by the school doctor and team as part of this service evaluation.

Results 1 family and 2 students refused. 2 parents concerned about appetite.

Teachers correctly identified $13 / 29$ students as having a problem with their weight.

$76 / 79$ students assessed. BMI range 15 to 50. 30/76 (35\%) met criteria for referral to special needs dietician, increasing service yearly caseload by a third. $27 / 76(35 \%)$ were $\geq 91^{\text {st }}$ BMI centile. $13 / 76(17 \%)$ were $\geq 98^{\text {th }}$ centile (see Figures 1 and 2 ).
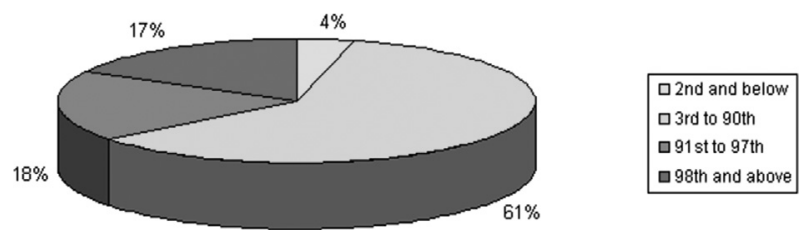

Abstract G465(P) Figure 1 BMI centiles

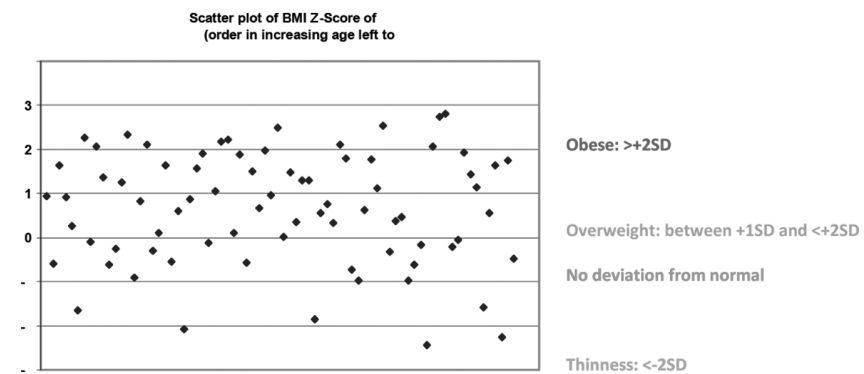

Abstract G465(P) Figure 2 WHO: A simple guide to classifying body mass index in children 2011 
There was no local Tier 2 or 3 weight management services available for this age group.

The school doctor action plan included education of teachers on obesity and introducing Tier 2 lifestyle weight management programme as pilot for secondary students. The special needs dietician negotiated a reduction of puddings. The dietetics service commissioners were informed of the need, resulting in plans to expand the service.

Conclusions There were many overweight students previously unidentified that required further management. Teachers did not accurately identify students that are overweight and therefore might not be best placed to initiate referrals. There are gaps in school health service including screening for obesity and services that should be in place for this age group according to NICE guidance. In today's obesogenic environment the school doctor role should include advocating for students to attain healthy weight, (Measuring Up 2013).

Acknowledgments School nurses, Teachers, Kitchen Staff, Dietician, Students and Parents, Tier 2 Team

\section{G466(P) PERCUTANEOUS ENDOSCOPIC GASTROSTOMY (PEG) IN DISABLE CHILDREN - A BENCH MARKING EXERCISE}

SS Saran, DW Wasala, NA Ayub. Paediatrics, Royal Shrewsbury Hospital, Shrewsbury, UK

\subsection{6/archdischild-2015-308599.420}

Background The Paediatric Gastroenterology Unit based at the Royal Shrewsbury Hospital provides a service for two hospitals. Its subsequent management is supported by the Community Paediatric Nurses.

A benchmarking exercise of the PEG Service was undertaken and audited against the European Society for Parenteral and Enteral Nutrition (ESPEN) Guidelines.

Methods All children with a PEG in-situ were identified from the Community Nurses PEG database. The elctronic records of these patients were accessed for relevant data and input into a Microsoft Excel 2010 database by a single researcher. The ESPEN standards were used to audit the service in the relevant domains.

Results 53 children with a PEG were identified. There was a clearly documented indication for a PEG in 96\%, with neurologically disabled children at risk of aspiration comprising the largest group (47\%). Feeding difficulties and Failure to thrive (FTT) made up 30\% while other indications were Sensory Feeding Disorder in combination with other primary disorders (13\%) dysmotility (4\%) and metabolic causes (2\%). The primary diagnosis was neurological in 55\%, gastrointestinal 11\%, renal $4 \%$ and cardiovascular, respiratory and endocrine $2 \%$ each. There were multiple diagnoses in $11 \%$

Nasogastric feeds were instituted and documented prior to the PEG in $94 \%$ and may have been used in a further $2 \%$ but not documented.

More then half the children had their PEG sited at the age of 13-36 months (52\%), a further 15\% under 13 months while $20 \%$ were after the age of 36 months. Almost one third of the children (29\%) had no complications from the PEG. Localised infection was the commonest complication (28\%) with granulation tissue (13\%), mechanical problems (dislodgement $8 \%$, blockage $4 \%$ ), skin ulceration $(10 \%)$ and leakage $(8 \%)$ as other complications.
PEG feeds were succesful in improving the weight centiles of these patients.

Parental satisfaction with the service could not be evaluated retrospectively.

Conclusions The PEG Service at the Shrewsbury and Telford Hospitals NHS Trust adheres to ESPEN standards in the majority of patients and is associated with a low complication rate. Parental Satisfaction with the service should be sought prospectively.

\section{G467(P) AUDIT OF THE QUALITY OF MEDICAL REPORTS FOR CHILDREN WITH SPECIAL EDUCATIONAL NEEDS}

${ }^{1} \mathrm{~A}$ Goodson, ${ }^{2} \mathrm{~T}$ Juniper, ${ }^{2} \mathrm{C}$ Lloyd, ${ }^{2} \mathrm{H}$ Miller, ${ }^{2} \mathrm{P}$ Reid, ${ }^{1} \mathrm{M}$ Rose. ${ }^{1}$ Department of Community Paediatrics, Oxford University Hospitals Trust, Oxford, UK; ${ }^{2}$ Medical School, Oxford University, Oxford, UK

\subsection{6/archdischild-2015-308599.421}

Introduction The purpose of the statutory assessment of a child's Special Educational Needs (SEN) is to gainclear picture of the whole child. Medical advice must be included and should describe any health provision reasonably required by the child's learning difficulties or disabilities. Families are now involved in the co-production of their child's Education Health and Care plan. For medical advice to be effective, it needs to be clear, accessible and specific, and ideally written in layman's terms.

Aim To assess the quality of the medical reports submitted to education for Statements of Special Educational Needs.

Method We audited all the medical reports submitted to education between January and June 2014 for a Statement of Special Educational Needs (SSEN). This work was part of pilot exploring data collection on child disability for planning purposes. A limited number of quality indicators were selected from medical advice guidelines. Data on coexisting health needs and actions arising were also recorded.

Results A total of 125 reports were analysed for children, median age 8.8 years, $74 \%$ boys. $48 \%$ of the reports were derived from existing medical records. $52 \%$ of reports were generated after a new medical consultation. Results from this latter group are presented.

$85 \%$ reports recorded growth, $85 \%$ vision, $72 \%$ hearing and $65 \%$ all three. Many medicals made reference to a lack of background information, especially for children with SEMH, ASD/ ADHD.

Advice related to the child's SEN and to associated medical problems (such as asthma) was not written consistently.

Many associated health problems were identified: 11\% of children were overweight, $18 \%$ obese. $17 \%$ had visual problems, $14 \%$ nutritional, $15 \%$ continence, $11 \%$ general medical problems. In many instances these problems were severe enough to prompt referral to therapy services for $9 \%$, to CAMHS $8 \%$, continence 5\%, general medical 9\%.

Conclusions The quality and detail of the medical reports was very variable and the medical examination identified many unmet health needs that might otherwise not have been recognised. These findings will be used to inform the crafting of medical advice and the development of new tools and guidelines for EHC plans. 